\title{
Breaking the diffusion limit with super-hydrophobic delivery of molecules to plasmonic nanofocusing SERS structures
}

\author{
F. De Angelis ${ }^{1,2}$, F. Gentile ${ }^{1,2}$, F. Mecarini', G. Das', M. Moretti', P. Candeloro², M. L. Coluccio ${ }^{1,2}$, \\ G. Cojoc ${ }^{2}$, A. Accardo1,2, C. Liberale', R. P. Zaccaria1, G. Perozziello1, L. Tirinato², A. Toma1, G. Cuda², \\ R. Cingolani ${ }^{1}$ and E. Di Fabrizio, ${ }^{1,2 \star}$
}

The detection of a few molecules in a highly diluted solution is of paramount interest in fields including biomedicine, safety and eco-pollution in relation to rare and dangerous chemicals. Nanosensors based on plasmonics are promising devices in this regard, in that they combine the features of high sensitivity, label-free detection and miniaturization. However, plasmonic-based nanosensors, in common with general sensors with sensitive areas on the scale of nanometres, cannot be used directly to detect molecules dissolved in femto- or attomolar solutions. In other words, they are diffusionlimited and their detection times become impractical at such concentrations. In this Article, we demonstrate, by combining super-hydrophobic artificial surfaces and nanoplasmonic structures, that few molecules can be localized and detected even at attomolar $\left(10^{-18} \mathrm{~mol} \mathrm{I}^{-1}\right)$ concentration. Moreover, the detection can be combined with fluorescence and Raman spectroscopy, such that the chemical signature of the molecules can be clearly determined.

arly diagnosis in medicine is a field in which nanotechnology can sensibly offer important and unprecedented benefits. For example, although it is well understood that blood contains a number of molecules and biomarkers that may reveal the presence of a disease, their identification or recognition is still challenging because of their very low concentrations. There is therefore an urgent need for novel devices with (i) superior sensing capabilities and/or (ii) increased spatial localization.

In the recent past, the combination of plasmonics and Raman spectroscopy has led to extraordinary success in few-molecule investigations by concentrating optical radiation energy in hot spots with areas of just a few $\mathrm{nm}^{2}$ (refs 1-4). Such electric-field hot spots are active only in the near-field region, that is, within a few nanometres of the surface. Unfortunately, in most practical applications, molecules are dispersed in solutions and are free to diffuse into the liquid volume, far from the plasmonic sensitive surfaces. Largearea sensors provide a high geometrical cross-section, but very poor signal-to-noise ratio when only few molecules are investigated (only a small portion of the sensitive area is actively involved). On the other hand, the reduction of the sensitive area down to few $\mathrm{nm}^{2}$ renders an encounter of the molecules and nanosensor very unlikely. It has been demonstrated ${ }^{5,6}$ that when the concentration of the solution is close to femtomolar and the linear size of the sensor is in the range of a few tens of nanometres, the accumulation time for the detection of a few molecules is on the scale of days. In other words, nanosensors cannot be used for low-concentration detection (below picomolar concentrations) because the accumulation time is far beyond practical timescales. Different approaches have been investigated to overcome these limitations, for example, by using plasmonic nanoparticles that can diffuse through the solution and meet the molecules of interest, microfluidic channels that drive the molecules towards the sensitive surfaces, and membrane nanopores acting as sieves that force molecules to pass through them ${ }^{7,8}$. Despite the great success of these techniques, the 'diffusion limit' still prevents their efficient exploitation in the femto/attomolar range.

Regardless of the detection technique applied, the challenge to be met is to find a way to drive molecules towards sensitive areas, or in other words, overcome the diffusion limit. In this Article, for the first time, surface nanostructuring is used to redesign and fabricate new and highly sensitive sensors in which super-hydrophobic surfaces and plasmonics nanostructures are combined in a synergistic way to allow single-molecule detection in highly diluted solutions, even at femto- or attomolar $\left(10^{-15} / 10^{-18} \mathrm{~mol} \mathrm{l}^{-1}\right)$ levels. Superhydrophobic surfaces allow us to drive and concentrate molecules over the sensing nano-area, where plasmonic electric-field hot spots are used to carry out molecule detection. The diffusion limit can then be overcome and time accumulation reduced to a few minutes, even for concentrations at attomolar levels.

We first show how a super-hydrophobic surface can be used to localize molecules in highly diluted solutions at a specific position. A set of spectroscopic experiments, based on fluorescence and Raman scattering, will be presented to demonstrate the extreme sensing capability and wide applicability of our method. Finally, an advanced plasmonic nanosensor expressly conceived to fully exploit the advantage of our approach will be presented.

\section{Device design and working principle}

Super-hydrophobicity ${ }^{9-11}$ is a phenomenon in which a drop placed on a surface adopts a quasi-spherical shape with a contact angle greater than $150^{\circ}$, rather than spreading or wetting indefinitely the plane of contact (Supplementary Movie M1). The present work takes advantage of the possibility of designing superhydrophobic surfaces with high contact angles and low friction forces (low friction coefficient), independent of the radius of the drop. Figure 1 shows that, when a drop of an extremely diluted 
a
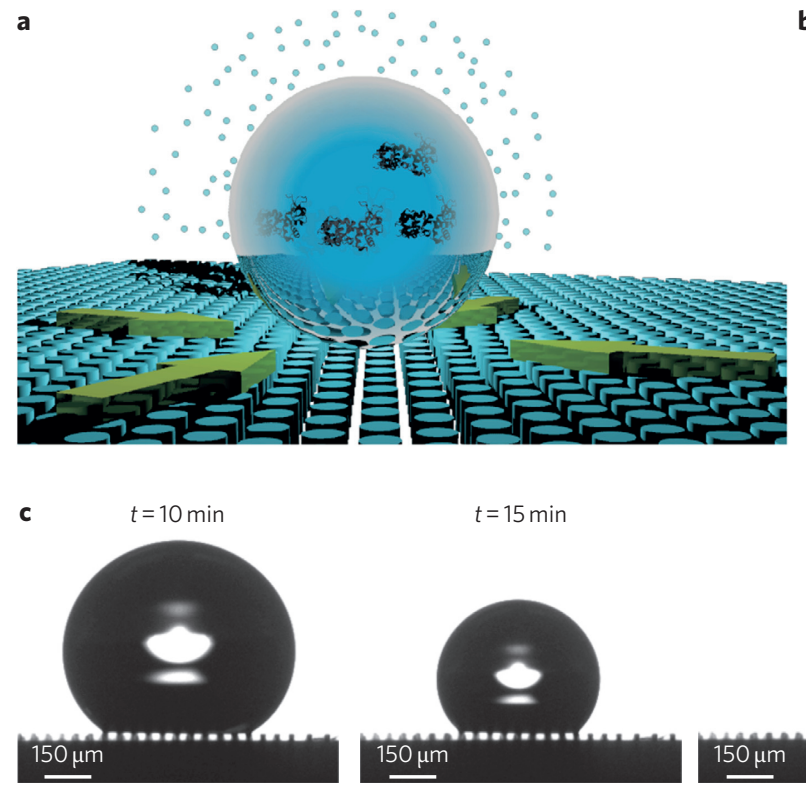

$t=15 \mathrm{~min}$

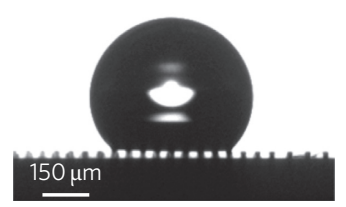

b

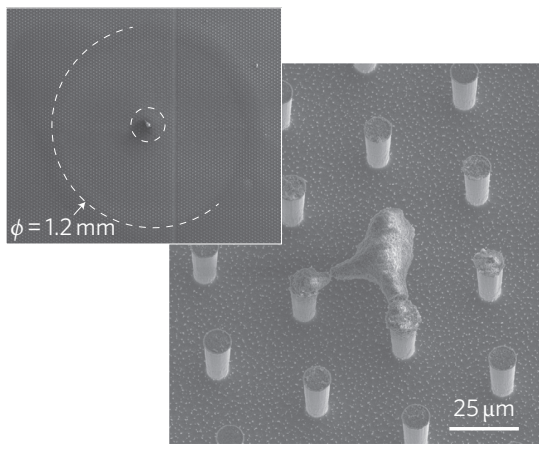

$t=25 \min$

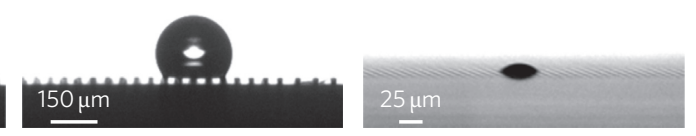

Figure 1 | High contact angle and evaporation process. a, Sketch representing the high contact angle and evaporation process with no pinning of the drop and no solute left on the substrate during drop concentration. b. SEM images of the footprint diameter of the drop and the suspended deposition of the solute on pillars. Notice that the whole content of the drop, with an initial contact area of $1.2 \mathrm{~mm}$ (original diameter, $2 \mathrm{~mm}$ ), is localized on a triangle with lateral sides of $\sim 25 \mu \mathrm{m}$. c, Contact angle measurements during evaporation at four different times (optical images taken with a microscope contact angle of $90^{\circ}$ geometry), showing the sliding and evaporation mechanism of the drop on the super-hydrophobic surface. Note that the drop slips on the surface, keeping the contact angle and the shape of the drop constant. The last image shows the collapsing condition (optical image taken at lower magnification to better show the volume reduction; see also Supplementary Movies M1 and M2).

solution is deposited on a textured, super-hydrophobic substrate and is allowed to evaporate, the drop will reduce in volume while maintaining its quasi-spherical shape (self-similar geometrical transformation). This behaviour is a result of the low adhesion forces between the drop and the surface; during evaporation, these allow the drop to slide on the surface, thereby avoiding it being pinned at its initial contact point ${ }^{12-15}$. During evaporation, the solution therefore becomes more and more concentrated. At the end of the process, when the shape and concentration reach a condition of instability, the drop collapses and the solute deposits in a suspended confined region with an area of a few square micrometres (Supplementary Movie M2). When designed properly (for details on surface design and its calculations, see Supplementary Section 1 ), the precipitation region and the sensitive area of the nanosensor are coincident and the detection of a few molecules (or a single molecule) is possible, even for solutions with initial concentrations on an attomolar scale.

Figure 2 presents scanning electron microscopy (SEM) images of super-hydrophobic surfaces made of silicon micropillar arrays with a typical periodicity of $30 \mu \mathrm{m}$, duty cycle (fraction of air to solid) of $\sim 3: 1$ and aspect ratio of $\sim 10$. Through an appropriate combination of micro- and nanofabrication (Supplementary Section 2), superhydrophobic surfaces and plasmonic nanosensors can be realized on the same chip. In this work, we fabricated different kinds of plasmonic nanostructures on the top of a silicon micropillar, including simple rough silver surfaces (Fig. 2b), regular arrays of silver nanoparticles (Fig. 2c), silver nanocones with circular grating coupler (Fig. 2d), and an advanced plasmonic nanosensor fully embedded in the micropillar array, which will be described in detail later (Fig. 2e).

\section{Device functions}

As mentioned above, the functions of these devices include (i) the concentration and localization of an initially greatly diluted solute into a very small region of the plane and (ii) the generation and accumulation of plasmon polaritons in the region of deposition to allow a few-molecule investigation by means of Raman scattering $^{16-24}$. The interest in this spectroscopy is related to its ability to provide a clear physical and chemical insight into the molecules under study. In the following, we report the use of such micronano hybrid devices to detect various molecules and biomolecules (rhodamine, lambda DNA, lysozyme) starting from solutions with concentrations ranging from femto- to attomolar. The geometries used were chosen for having stable hydrophobic conditions and appropriate plasmonic structure. In Supplementary Fig. S1.6, the contact angle $\theta_{\mathrm{e}}^{\mathrm{c}}$ is reported as a function of pillar diameter $d$ and distance $\delta$ for the hexagonal lattice. In particular, it is shown that a region of stability is achieved when a contact angle between $160^{\circ}$ and $170^{\circ}$ is obtained for a wide range of $d$ and $\delta$ between tens of nanometres and a few micrometres. We note here that super-hydrophobic surfaces are reproducible and can be scaled up for high-throughput production using micro- and nanofabrication techniques. Moreover, independent of the analytical technique used (fluorescence, Raman, and other spectroscopies or techniques), they provide an easy and fast concentration and localization method fully compatible with existing protocols in biology and medicine.

\section{Detection of rhodamine at femto- to attomolar concentrations} As an introductory experiment to show the potential of this procedure, we report the detection of rhodamine from water solutions below a concentration of $1 \mathrm{fM}$ (see Supplementary Section 3 for sample preparation). Evaporation of the drop (on bare superhydrophobic surfaces, Fig. 2a) was followed over time until precipitation of the solute. The solute molecules were confined into a small area of a few tens of micrometres square. Solutions of progressively decreasing concentration were investigated. Figure 3 a presents an SEM image of the residual solute of rhodamine $6 \mathrm{G}$ at the end of a process of evaporation starting from a drop with a diameter of $2 \mathrm{~mm}$. Notice the strong concentration and localization of rhodamine, which precipitates onto a small area bridging the pillars. 

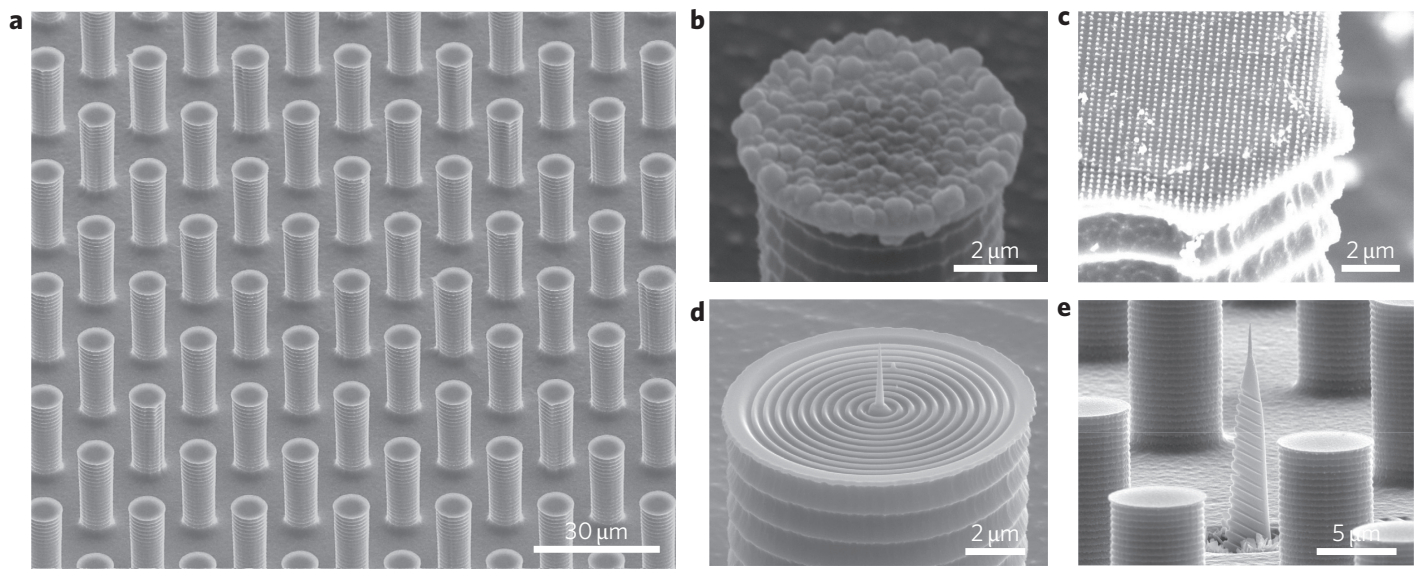

Figure 2 | Architecture of the four different devices fabricated in this work. a, Periodical array designed to have a high contact angle $\left(>150^{\circ}\right)$ and high drop stability. $\mathbf{b}$, The first type of pillar, with its top decorated with silver nanoparticles applied by electroless deposition. Random nanoparticle deposition is the simplest way of combining super-hydrophobicity and plasmonic enhancement. c, The second type of pillar, with the plasmonic nanostructures arranged in a regular array. d. The plasmonic structure represented by a nanocone coupled with a circular grating for efficient generation of plasmon polaritons on the cone. e, The plasmonic structure embedded in the super-hydrophobic array, with a single pillar used for plasmonic enhancement and detection.
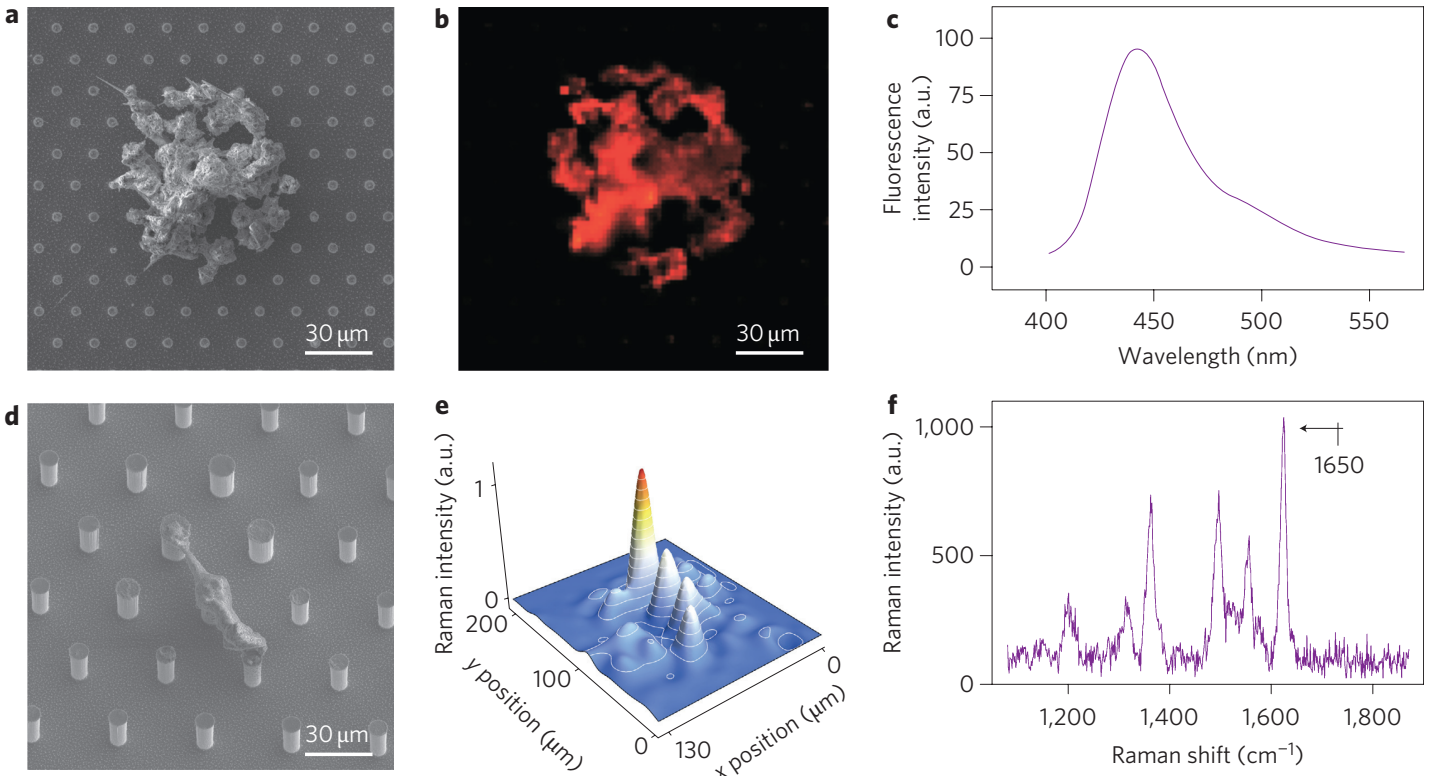

Figure $\mathbf{3}$ | Localization and spectroscopic measurements. a, SEM image of solute precipitation from a $1 \mathrm{fM}$ solution of rhodamine. $\mathbf{b}, \mathbf{c}$, Fluorescence measurements of rhodamine (b) and its spectral signature (c). d, SEM image of solute precipitation from a 10 aM solution of rhodamine. e,f, Raman mapping measurement of rhodamine (e) and its spectral signature (f).

In each experiment the residual solute was localized in a region with a linear extension smaller than a few tens of micrometres. In the case presented in Fig. 3a, the initial concentration of rhodamine was $1 \times 10^{-15} \mathrm{M}$. The fluorescent image in Fig. $3 \mathrm{~b}$ confirms the identification of the residue as rhodamine, and it is clear that no appreciable quantity is left around the precipitate on the neighbouring pillars. To further substantiate the method for concentrations as low as $10 \mathrm{aM}\left(10^{-17} \mathrm{M}\right)$, micro-Raman mapping measurements were performed using the device of Fig. $2 b$. The results are reported in Fig. 3d-f. The intensity and contrast of the Raman signal are very strong due to the presence of the silver nanostructures. A mapping analysis (Fig. 3e) was performed, with detection of the band centred at $1,650 \mathrm{~cm}^{-1}$, and overlaps clearly with the SEM image of Fig. $3 \mathrm{~d}$, in which the entire precipitate is seen to lie on only three pillars.
Considering that the quantity of solution initially deposited was $\sim 20 \mu \mathrm{l}$ (initial drop diameter, $\sim 2 \mathrm{~mm}$, containing R6G molecules), these devices reveal or detect $\sim 100$ molecules, starting from attomolar concentrations, with relative ease of use. We note that when the same drop was deposited on a conventional flat plasmonic substrate, the drop expanded over the metal surface and, at the end of evaporation, R6G molecules were deposited over an area of $\sim 5 \times 5 \mathrm{~mm}^{2}$. This indicates a concentration factor of at least $1 \times 10^{4}$ $\left(5 \times 5 \mathrm{~mm}^{2} / 50 \times 50{\mu \mathrm{m}^{2}}^{2}\right)$ between the two arrangements.

\section{Single lambda DNA molecule from attomolar concentration} Lambda DNA molecules were chosen as a test system for the recognition and localization of single molecules because of their clear and unambiguous imaging results. Lambda DNA solutions were 

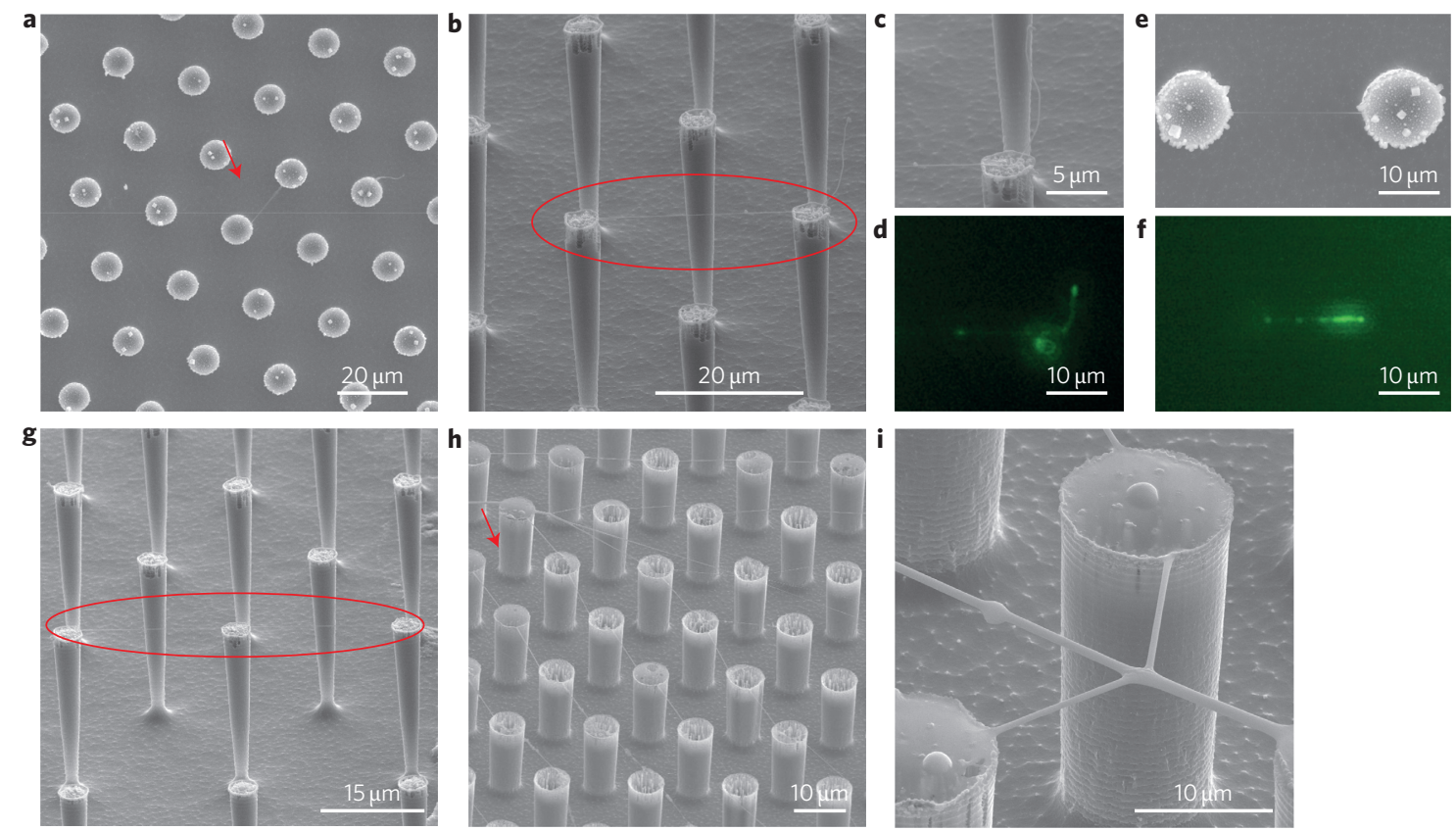

Figure 4 | Single lambda-DNA localization and detection from a 10 aM solution. a, SEM image showing a single molecule of DNA on an area of $\sim 150 \times$ $150 \mu \mathrm{m}^{2}$. b,c,e, SEM images showing a single suspended DNA molecule from different viewpoints. d,f, Optical fluorescent measurements of the single DNA molecule. $\mathbf{g}$, Two lambda-DNA bridges are localized. $\mathbf{h}$, A network is obtained by increasing the lambda-DNA concentration in the starting solution. $\mathbf{i}$, Detail of a suspended node as a result of a few lambda-DNA molecule interaction.

prepared following a staining and serial dilution protocol (Supplementary Section 3). Preliminary experiments demonstrated that a double-stranded lambda DNA molecules (48,502 bp) can be deposited, suspended and stretched between two or more superhydrophobic pillars (Supplementary Section 4). As a result of surface super-hydrophobicity, dewetting of the DNA-containing solution occurred on the top surface, allowing the DNA to link exactly on the top of the pillars. An attomolar concentration of DNA stained with YOYO-1 dye (Supplementary Section 3) in a $3 \mu \mathrm{l}$ drop was evaporated on the substrates for 20 min (a $3 \mu l$ drop with a concentration of $1 \times 10^{-18} \mathrm{~mol}^{-1}$ contains, statistically, two DNA molecules). Samples were analysed both in epifluorescence optical and SEM microscopy. The results are shown in Fig. 4. Fluorescent DNA was clearly visible (Fig. 4d,f). SEM images of the same sample showed a correspondence at the same position with the fluorescent DNA stretches (Fig. 4b,c,e). The filaments were 10-30 $\mu \mathrm{m}$ long. From Fig. $4 \mathrm{~b}$, we found that filaments longer than $15 \mu \mathrm{m}$ were probably due to DNA overlapping. In other words, unpaired overlapping of lambda filaments makes a longer filament. Moreover, in a more accurate observation in SEM microscopy the filament appears to have two distinct diameters. In Fig. 4a, a wider SEM snapshot over an area of $\sim 150 \times 150 \mu \mathrm{m}^{2}$ does not reveal the presence of other DNA molecules, which is consistent with the nominal concentration of $1 \times 10^{-18} \mathrm{~mol} \mathrm{l}^{-1}$. This concentration and localization process can be accurately controlled and, by varying the dilution, it is possible to deposit an increasing number of molecules, as in Fig. $4 \mathrm{~g}$, and create a suspended regular lambda DNA network, as shown in Fig. 4h,i (Supplementary Section 4).

We envisage that the devices we have presented, prepared entirely without any chemical modification of the DNA and isolated from interaction with the substrate, could be used in wide range of applications. These might include the design of DNA wires for DNA-template electronic devices ${ }^{25}$, the positioning and study of protein interactions and enzyme cascading ${ }^{26}$, the rational assembly of nanoarrays ${ }^{27,28}$, for structuring live cell friendly immobilization beads ${ }^{29}$, as a building base for the design of DNA hydrogels for cell-free protein expression ${ }^{30}$, or even to study DNA motors or walkers $^{31}$ to explore the design of drug transport and release.

\section{Lysozyme molecules by advanced plasmonic devices}

As introduced above, super-hydrophobic substrates and plasmonic structures can be designed independently. In other words, given the pillar architecture, which is designed in the stable superhydrophobic region (Supplementary Section 1), it is possible to choose separately the textures covering the pillars that locally enhance the electric field. In recent years, plasmonic tips and antennas made of noble metals have led to few-molecule investigations using Raman spectroscopy ${ }^{32-36}$. To fully exploit the advantage of our approach, we designed super-hydrophobic surfaces with a plasmonic cone directly embedded in a micropillar array. As shown in Fig. 5, the cone was fabricated at the centre of the array as a replacement for a micropillar. For symmetry reasons, the cone can be viewed as a spatial defect that breaks the spatial symmetry defined by the pillars. As a result, the hydrophobic radial forces cause the collapse of the drop on the cone tip (Supplementary Section 1). In other words, during evaporation, such a local defect drives the drop towards the tip itself, as a result of the reduced hydrophobic effect. As a consequence, molecules initially dispersed in solution will be progressively guided towards the plasmonic tip, where they will be deposited at the end of the evaporation process.

The plasmonic tip, consisting of a silver nanocone with a grating on one side, was fabricated by focused ion beam milling and electron-beam-induced deposition (see Supplementary Section 2 for details). Device simulation was performed with commercial software (www.lumerical.com) using a finite-difference time-domain (FDTD) approach. The results are shown in Fig. 5e (see Supplementary Section 5 for details). The lateral grating was designed to provide an efficient coupling (even if the symmetry of the plasmonic mode in the cone was not fully fulfilled) between the incoming laser $(\lambda=532 \mathrm{~nm})$ and the nanocone. Surface plasmon polaritons are launched from the grating ( $480 \mathrm{~nm}$ period) towards the tip, where electric-field enhancement occurs. For a tip with a $10 \mathrm{~nm}$ radius of curvature, the calculated electric-field 

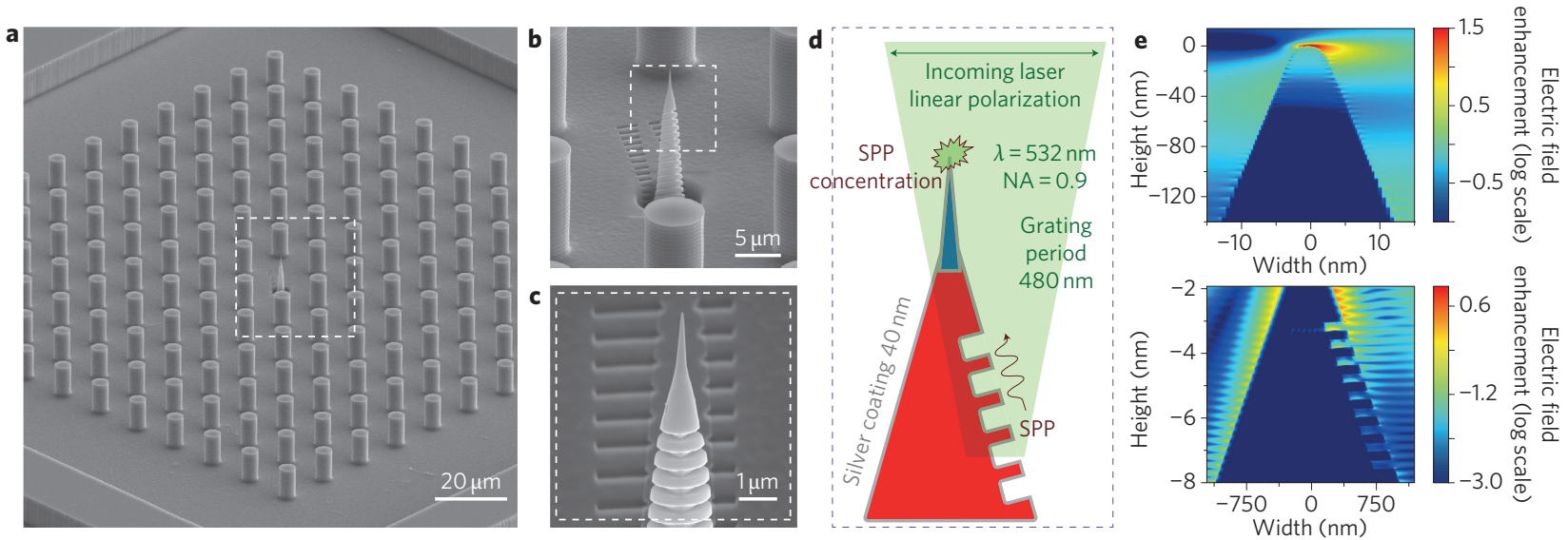

Figure 5 | Super-hydrophobic nanostructure. a, Super-hydrophobic device with embedded plasmonic nanostructure. b,c, Details of the device. d. Measurement principle. After evaporation, the solute precipitates on the tip of the nanocone. Through a scanning procedure an optimal illumination/detection of the grating/sample is obtained. e, FDTD simulation results, showing an asymmetrical intensity field, in good agreement with the experimental imaging measurements reported in the Fig. 6b.
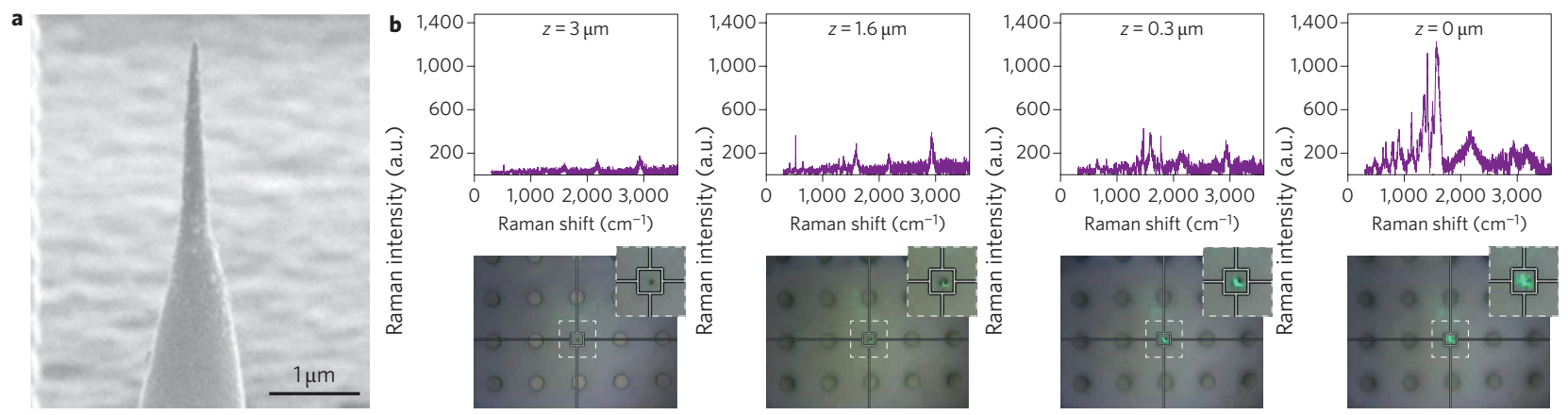

Figure 6 | Super-hydrophobic device with embedded plasmonic nanostructure. a, SEM image after lysozyme precipitation. b, Optical images and corresponding Raman measurements while scanning along the optical axis. Optimal conditions are reached at $z=0 \mu m$, where a lysozyme high-contrast Raman signal is obtained.

enhancement is $\sim 35$ and the Raman enhancement is $1.5 \times 10^{6}$, strong enough for the detection of a few molecules located in the proximity of the tip end. In Fig. $6 \mathrm{~b}$ (bottom), activation of a surface plasmon polariton is clearly shown when the illumination and imaging of the grating/nanocone are both properly satisfied $(0 \leq z \leq 1.6 \mu \mathrm{m})$. In these conditions, the Raman spectra of lysozyme were simultaneously collected as described in the following.

A $160 \mathrm{nl}$ droplet was deposited on the surface of the device of Fig. 5 using a micro-injector system. The droplet contained lysozyme at a concentration of $1 \mathrm{fM}(\sim 100$ molecules in $160 \mathrm{nl}$ drops). At the end of the evaporation process (which lasted a few seconds in this case because the volume of the solution was $\sim 30$ times smaller than in previous experiments), lysozyme accumulated on the silver cone, as can be seen in Fig. 6a. Notice that the material along the cone surface includes additional environmental debris deposited in a dry condition after drop evaporation. As demonstrated in previous works ${ }^{32}$, only molecules deposited in close proximity to the tip are efficiently detected because the enhancement decreases very rapidly away from the apex. We estimate that, statistically and for geometrical reasons (the cone radius of curvature is $\sim 10 \mathrm{~nm}$ ), fewer than five molecules are deposited on the tip vertex (lysozyme can be described as a slightly deformed sphere with a diameter of $\sim 4 \mathrm{~nm}$; refs $37-40$ ). The spectra of the lysozyme are shown in Fig. $6 \mathrm{~b}$ as a function of $z$ scan position ( $z$ is the scan direction along the optical axis of the illuminating objective). For different positions of illumination/acquisition along the vertical direction ( $z$-axis), the Raman signal is observed to acquire a very high contrast when the coupling with the grating and the detection conditions are optimized (Supplementary Movie M3). In these conditions, the Raman sensitivity and spectra details increase strongly. Figure $6 \mathrm{~b}$ (bottom) clearly shows (as a function of $z$ scan) activation of the plasmon polaritons when optimal coupling/detection is reached.

The spectra taken for lysozyme when focused $3 \mu \mathrm{m}$ out of the optimal position shows very limited information about the substance, as only three weak bands are observed centred at $\sim 2,940$, 2,184 , and $1,600 \mathrm{~cm}^{-1}$, attributed to $\mathrm{C}-\mathrm{Hx},-\mathrm{S}-\mathrm{CHx}$ and aromatic bands (Phe, phenylalanine; Trp, tryptophan). As the focal point moves towards the optimal illumination $(z \leq 1.6 \mu \mathrm{m})$, various characteristic bands of lysozyme start appearing, providing rich chemical information about the protein (Fig. 6b, $z<3 \mu \mathrm{m}$ ). Vibrational bands are observed centred at 1,610 (Phe, Trp), 1,555-1,568 (amide II), 1,450 (C-Hx), 1,350 (Trp), 1,230-1,295 (Amide III), 1,069 and 1,126 (C-N stretching), 990 ( $\alpha$-helix), 895 (Trp), 650 (C-S stretching) and $620 \mathrm{~cm}^{-1}$ (Phe breathing), in addition to the broad band centred around $3,000 \mathrm{~cm}^{-1}(\mathrm{C}-\mathrm{Hx}$ stretching), which confirm the presence of proteins at the tip. Furthermore, the most significant band centred at $2,140 \mathrm{~cm}^{-1}$, attributed to the $-\mathrm{S}-\mathrm{CHx}$, can be clearly observed. This band is relatively less visible in the protein spectrum from the liquid sample. The broad band around $3,350 \mathrm{~cm}^{-1}$, which is related to the $\mathrm{N}-\mathrm{Hx}$ stretching (amine groups), as shown in Fig. 6b $(z=0)$, is also 
observed. Here, it should be noted that even if the scattering crosssection for $\mathrm{N}-\mathrm{H}$ vibration is very low, this band is observed in our measurement thanks to surface plasmon generation on the metal surface and thereafter the enhancement of the electric field on the nanocone tip.

\section{Conclusions}

In conclusion, the combination of super-hydrophobic surfaces and nanoplasmonics allows us to overcome the limit dictated by diffusion when sensors approach a nanoscale length. The present achievement combats the problem of detecting few molecules when the initial construct is highly diluted and the total solution volume lies between a few nanolitres and microlitres. When applied to biomedicine, the present results, combined with already available purification methods, suggest the possibility of improving the early detection of several diseases, including cancer, where the number of clinically significant molecules at the onset of the pathology is very small and often generated by a single cell.

\section{Methods}

Different fabrication methods were used for fabricating the present devices (see details in Supplementary Section 2). Optical lithography combined with deep reactive ion etching and electroless deposition was used for defining micropillars with random silver nanograins. Electron-beam lithography was used to fabricate micropillars decorated with regular arrays of silver nanodots. The micropillars, combined with plasmonic nanocones and gratings, were also fabricated using focused ion beam milling at an energy of $30 \mathrm{keV}$ and with an ion beam current between $500 \mathrm{pA}$ and $1 \mathrm{nA}$. The nanocone was grown on top of the silicon tapered pillar using electron-beam-induced deposition from a platinum-based gas precursor. The body of the cone was $\sim 10 \mu \mathrm{m}$ high, and the base diameter was $2 \mu \mathrm{m}$. Finally, a thin layer of silver $(40 \mathrm{~nm})$ was deposited on the device by means of thermal evaporation.

Received 9 June 2011; accepted 8 August 2011; published online 18 September 2011

\section{References}

1. Michaels, A. M., Nirmal, M. \& Brus, L. E. Surface enhanced Raman spectroscopy of individual rhodamine $6 \mathrm{G}$ molecules on large Ag nanocrystals. J. Am. Chem. Soc. 121, 9932-9939 (1999).

2. Stöckle, R. M., Suh, Y. D., Deckert, V. \& Zenobi, R. Nanoscale chemical analysis by tip-enhanced Raman spectroscopy. Chem. Phys. Lett. 318, 131-136 (2000)

3. De Angelis F. et al. A hybrid plasmonic-photonic nanodevice for label-free detection of a few molecules. Nano Lett. 8, 2321-2327 (2008).

4. Schuller, J. A. et al. Plasmonics for extreme light concentration and manipulation. Nature Mater. 9, 193-204 (2010).

5. Sheehan, P. E. \& Whitman, L. J. Detection limits for nanoscale biosensors. Nano Lett. 4, 803-807 (2005).

6. Nair, P. R. \& Alam, M. A. Performance limits of nanobiosensors. Appl. Phys. Lett. 88, 233120 (2006).

7. Lassiter, J. B. et al. Close encounters between two nanoshells. Nano Lett. 8, 1212-1218 (2008).

8. Fatemeh, E. et al. Nanoholes as nanochannels: flow-through plasmonic sensing. Anal. Chem. 81, 4308-4311 (2009).

9. Luo, M. S. C. et al. Artificial lotus leaf by nanocasting. Langmuir 21, 8978-8981 (2005).

10. Reyssat, M., Pépin, A., Marty, F., Chen, Y. \& Quéré, D. Bouncing transitions on microtextured materials. Europhys. Lett. 74, 306-319 (2006).

11. Accardo, A. et al. In situ X-ray scattering studies of protein solution droplets drying on micro- and nanopatterned superhydrophobic PMMA surfaces. Langmuir 26, 15057-15064 (2010).

12. McHale, G., Shirtcliffe, N. J. \& Newton, M. I. Super-hydrophobic and super-wetting surfaces: analytical potential? Analyst 129, 184-187 (2004).

13. Mahadevan, L. \& Pomeau, Y. Rolling droplets. Phys. Fluids 11, 2449-2454 (1999).

14. Aussillous, P. \& Quéré, D. Liquid marbles. Nature 411, 924-927 (2001).

15. McHale, G., Aqil, S., Shirtcliffe, N. J., Newton, M. I. \& Erbil, H. Y. Analysis of droplet evaporation on a superhydrophobic surface. Langmuir 21, 11053-11060 (2005).

16. Grand, J. et al. Role of localized surface plasmons in surface-enhanced Raman scattering of shape-controlled metallic particles in regular arrays. Phys. Rev. B, 72, 033407 (2005).

17. Stockman, M. I. Nanofocusing of optical energy in tapered plasmonic waveguides. Phys. Rev. Lett. 93, 137404 (2004).

18. Chen, X. W., Sandoghdar, V. \& Agio, M. Highly efficient interfacing of guided plasmons and photons in nanowires. Nano Lett. 9, 3756-3761 (2009).
19. Lindquist, N. C., Nagpal, P., Lesuffleur, A., Norris, D. J. \& Oh, S. H. Three dimensional plasmonic nanofocusing. Nano Lett. 10, 1369-1373 (2010).

20. Genet, C. \& Ebbesen, T. W. Light in tiny holes. Nature 445, 39-46 (2007)

21. Bargioni, A. W. et al. Hyperspectral nanoscale imaging on dielectric substrates with coaxial optical antenna scan probes. Nano Lett. 11, 1201-1207 (2011)

22. Genov, D. A., Sarychev, A. K., Shalaev, V. M. \& Wei, A. Resonant field enhancement from metal nanoparticle arrays. Nano Lett. 4, 153-158 (2004).

23. Neacsu, C. C. et al. Near-field localization in plasmonic superfocusing: a nanoemitter on a tip. Nano Lett. 10, 592-596 (2010).

24. De Angelis, F., Liberale, C., Coluccio, M. L., Cojoc, G. \& Di Fabrizio, E. Emerging fabrication techniques for $3 \mathrm{D}$ nano-structuring in plasmonics and single molecule studies. Nanoscale 3, 2689-2696 (2011).

25. Braun, E., Eichen, Y., Sivan, U. \& Ben-Yoseph, G. DNA-templated assembly and electrode attachment of a conducting silver wire. Nature 391, 775-778 (1998).

26. Niemeyer, C. M. Semisynthetic DNA-protein conjugates for biosensing and nanofabrication. Angew. Chem. Int. Ed. 49, 1200-1216 (2010).

27. Seeman, N. C. Feature DNA in a material world. Nature 421, 427-431 (2003)

28. Yan, H., Park, S. H., Finkelstein, G., Reif, J. H. \& LaBean, T. H. DNA-templated self-assembly of protein arrays and highly conductive nanowires. Science 301, 1882-1884 (2003)

29. Schroeder, H., Ellinger, B., Becker, C. F., Waldmann, H. \& Niemeyer, C. M. Generation of live-cell microarrays by means of DNA-directed immobilization of specific cell surface ligands. Angew. Chem. Int. Ed. 46, 4180-4183 (2007).

30. Park, N., Um, S. H., Funabashi, H., Xu, J. \& Luo, D. A cell-free protein producing gel. Nature Mater. 8, 432-437 (2009).

31. Sherman, W. B. et al. A precisely controlled DNA biped walking device. Nano Lett. 4, 1203-1207 (2004).

32. De Angelis, F. et al. Nanoscale chemical mapping using three-dimensional adiabatic compression of surface plasmon polaritons. Nature Nanotech. 5, 67-72 (2010)

33. Fang, Y., Seong, N. H. \& Dlott, D. D. Measurement of the distribution of site enhancements in surface-enhanced Raman scattering. Science 321, 388-392 (2008).

34. Rodrìguez-Lorenzo L. et al. Zeptomol detection through controlled ultrasensitive surface-enhanced Raman scattering. J. Am. Chem. Soc. 131, 4616-4618 (2009)

35. Taminiau, T. H., Stefani, F. D., Segerink, F. B. \& Van Hulst, N. F. Optical antennas direct single-molecule emission. Nature Photon. 2, 234-237 (2008).

36. Das, G. et al. Nano-patterned SERS substrate: application for protein analysis vs. temperature. Biosens. Bioelectron. 24, 1693-1699 (2009).

37. Kendrew, J. C. et al. A three-dimensional model of the myoglobin molecule obtained by X-ray analysis. Nature 181, 662-666 (1958).

38. Revenis, M. E. \& Kaliner, M. A. Lactoferrin and lysozyme deficiency in airway secretions: association with the development of bronchopulmonary dysplasia. J. Pediatr. 121, 262-270 (1992).

39. Lundberg, D., Carnerup, A. M., Schillén, K., Miguel Mda, G. \& Lindman B. Phase behavior and coassembly of DNA and lysozyme in dilute aqueous mixtures: a model investigation of DNA-protein interactions. Langmuir 26, 2986-2988 (2010).

40. Lönnerdal, B. Nutritional and physiologic significance of human milk proteins Am. J. Clin. Nutr. 77, 1537-1543 (2003).

\section{Acknowledgements}

The authors thank L. Fruk (Karlsruhe Institute of Technology) for discussions and suggestions on biological aspects of this work, and R. La Rocca, R. Tallerico and A. Nicastri (BIONEM University of Magna Graecia) for sample preparation. This work was funded under European Project SMD FP7-NMP 2800-SMALL-2 (proposal no. CP-FP 229375-2), Project NANOANTENNA FP7-HEALTH-2009 (grant agreement no. 241818), Italian project FIRB 'Rete Nazionale di Ricerca sulle Nanoscienze ItalNanoNet' (cod. RBPR05JH2P_010).

\section{Author contributions}

F.D.A. conducted FIB milling, electron-beam deposition and numerical simulations. F.G. carried out micropillar fabrication, super-hydrophobic measurements and modelling. F.M. prepared samples and carried out evaporation and sputtering. G.D. and P.C. conducted Raman measurements. M.M. carried out DNA deposition and protocol optimization. M.L.C. carried out electroless deposition and protocol optimization. G.C., A.A., L.T. and A.T. were involved in super-hydrophobic characterization. C.L. carried out fluorescence measurements. R.P.Z. conducted electromagnetic modeling. G.P. was responsible for superhydrophobic and microfluidic design. G.C. carried out the biological overview and protein evaluation. R.C. was responsible for project planning. E.D.F. was proposer and project coordinator.

\section{Additional information}

The authors declare no competing financial interests. Supplementary information accompanies this paper at www.nature.com/naturephotonics. Reprints and permission information is available online at http://www.nature.com/reprints. Correspondence and requests for materials should be addressed to E.D.F. 